\title{
Ward management practice in Gastrointestinal Surgical Ward During the COVID-19 Epidemic
}

\author{
XIU YING ZHANG ${ }^{1}$, Liren Wang ${ }^{1}$, Shuguang $\mathrm{Li}^{2}$, and Hong Jiang ${ }^{3}$ \\ ${ }^{1}$ Affiliation not available \\ ${ }^{2}$ Huashan Hospital Fudan University \\ ${ }^{3}$ Huashan Hospital Affiliated to Fudan University
}

April 28, 2020

\begin{abstract}
Objective During the epidemic of COVID-19, efficient ward management is an important part of preventing nosocomial infection while ensuring the medical works properly without any Covid-19 case. Method Ward management practice was updated, including optimizing the personnel access processes and strengthening infection control procedures in the gastrointestinal surgery ward during Jan. 23 to Mar. 15, 2020. Results With 43 beds open, 192 patients were treated, 72 were operated on, 89 received chemotherapy after surgery, and 187 were discharged from hospital. All patients achieved the expected recovery, and none of these patients, their families,or medical staff were infected. Conclusion Ward management based on low and guidelines during the COVID-19 epidemic is an effective measure to limit infection spread within the hospital and to healthcare workers.
\end{abstract}

\section{INTRODUCTION}

Currently, coronavirus disease 2019 (COVID-19) poses a significant threat to global health. World Health Organization (WHO) has declared this outbreak as a "public health emergency of international concern" on January 31, 2020.11World Health Organization. WHO Director-General's opening remarks at the media briefing on COVID-19 -11 March 2020. Geneva: World Health Organization; 2020Within the first two months of the outbreak, the epidemic spread rapidly around the country and the world. it was inferred that the nosocomial infection rate could reach $41 \%$ without rapid intervention.22Wang D, Hu B, Hu C, Zhu F, Liu X, Zhang J, Wang B, Xiang H, Cheng Z, Xiong Y, et al. Clinical Characteristics of 138 Hospitalized Patients With 2019 Novel Coronavirus-Infected Pneumonia in Wuhan, China. JAMA. 2020 Feb 7. doi: 10.1001/jama.2020.1585. In the absence of vaccines and specific treatment, the only available tools to control person-to-person transmittable diseases in healthcare settings are isolation, quarantine and infection control. Gastrointestinal surgical ward is characteristed by more emergency and more selective operations. How to balance emergency or outpatient patients and COVID-19 control has become a challenge to our ward. On the basis of the Chinese Law on the Prevention and Control of Infectious Diseases,33Standing Committee of the National People's Congress. Law on prevention and control of infectious diseases of the People's Republic of China (2013-6-29, Revised). China, Standing Committee of the National People's Congress, June 29, 2013. http://www.gov.cn/banshi/2005- 08/01/content_19023.htm. the Chinese Clinical Guideline for COVID-19 Diagnosis and Treatment (5th edition)44National Health Committee of the People's Republic of China. Chinese Clinical guideline for COVID-19 diagnosis and treatment (5th Edition). China, National Health Committee, February 4, 2020.http://www.nhc.gov.cn/yzygj/s7653p/202002/3b09b894ac9b4204a79db5b8912d4440.shtml. and Guideline of prevention and control of COVID-19 in medical institution (1st Edition),55National Health Committee of the People's Republic of China. Guideline of prevention and control of COVID-19 in medical institution (1st Edition). China, National Health Committee, January 22, 2020. http://www.nhc.gov.cn/yzygj/s7659/202001/b we updated the ward management rules and procedures, optimized the personnel access process and strength- 
ened infection control in our gastrointestinal surgery ward. And so far, our hospitalization rate reached $80 \%$ with zero infections among patients and medical staff, the following report is our ward management practice.

\section{METHOD}

\subsection{Manage Personnel Access to the Ward}

All people entering the ward, including medical staff, rear service personnel, patients and their family members were included in this epidemiological investigation. Passively screen all people for symptoms of acute respiratory illness before entering the healthcare facility every day and the results would be reported to the hospital protection department, which would be reviewed again by managers. Focus areas(especially areas in Hubei Province, ) were identified as areas with most potential risk of exposure to Coronavirus Disease 2019 (COVID-19) throughout the country. All patients and their family members from those areas were investigated and archived according to a new registration system. And only those without fever, respiratory symptoms or abnormal chest CT could be admitted to hospital.

Caregivers and visitors were restricted to reduce the flow and density of population and a fixed caregiver was allowed in our ward. Another substitutive caregivers if needed should be screened prior to entry to the unit, including epidemiological investigation, body temperature, and chest CT before admission. At the same time, the body temperature was monitored twice a day for the duration of their stay. The number of nurses and physician present during treatment were limited to only those essential for patient care and support. Establish visitor policy for monitoring and managing visitors. Limit visitors to our ward to only those essential for the patient's physical or emotional well-being and those with a temporary one-time pass issued by the head nurse, but if fever or COVID-19 symptoms were present, the visitor would not be allowed entry into the ward. All visitors must wear a facemask at limited visitation hours while in our ward and perform frequent hand hygiene. Visitors could enter and go out of the ward along the designated route during the specified period of time. Individual training program for all visitors on hand hygiene and appropriate PPE use before entering our ward was developed.

As part of routine practice, healthcare personnel were be asked to regularly monitor themselves for fever and symptoms of COVID-19, and only those without fever and symptoms of COVID-19 could remain to work. Flexible shift of On-the-job personnel were implemented to ensure the normal work.

\subsection{Strengthen infection control and block transmission routes}

We emphasized hand hygiene and provided supplies and instruction on respiratory hygiene, including alcoholbased hand rub (ABHR) with $75 \%$ alcohol, tissues, and no-touch receptacles for disposal, at the entrances of inpatient building and our ward. Instruction on hand hygiene, limiting surfaces touched, and use of personal protective equipment(PPE) according to current hospital policy were provided at every entrance. We updated the cleaning and disinfection procedures : using cleaners and water to pre-clean surfaces prior to applying an hospital-grade disinfectant to frequently touched surfaces and objects every day.

A separate and well-ventilated conversation room was specially set up and ventilated during the conversation. All the rooms underwent appropriate cleaning and surface disinfection before it is returned to use once again and records must be made to ensure all work done.

The level of healthcare personal protection(HCP) was determined according to the patient's condition and exposure risk to SARS-CoV-2. The primary protection including hand hygiene and facemask was performed before and after all patient contact, and when facing patients and family members with symptoms of COVID-19 or other respiratory infection (e.g., fever, cough), we provided supplies and equipment necessary for the consistent observance of Standard Precautions(second grade protection),11National Health Committee of People's Republic of China. Regulation for prevention and control of healthcare associated infection of airborne transmission disease in healthcare facilities. December 27, 2016. http://www.nhc.gov.cn/wjw/s9496/201701/7e0e8fc6725843a including hand hygiene products and personal protective equipment(PPE) (e.g.N95 respirators, gloves, gowns, face and eye protection). Adhere to standard precautions, appropriate PPE were selected according 
to PPE supply and the level of anticipated contamination. When available, N95 respirators are preferred which should be prioritized for situations where the care of patients with respiratory protection.

Once the patient conforms to any two of fever, respiratory symptoms and chest CT with covid-19 imaging characteristics, the contingency plan made at the begining of the COVID-19 is activated. The plan includes isolating symptomatic patients as soon as possible in a separate, well-ventilated room with door closed and private bathroom, disinfection and isolation of the room, healthcare personnel protection, et.al. Primary nurses were dedicated to care for patients during their shift to avoid the situation that multiple nurses carry out different nursing operations for the same patient. A special operating room would be arranged for such patients requiring emergency surgery, on the contrary elective procedures and surgeries would postponed.

Provide all healthcare personnel including but not limited to medical, nursing, clinical technicians, rear service personnel, patients and family members with job or task specific education and training on COVID19 and preventing transmission of infection, such as epidemiological investigation, hand hygiene and PPE. We enhanced timely education and training by applying multiform learning, combining online and offline education. Online education, such as Tencent Meeting Application and WeChat education became our main education approach. Group offline learning was eliminated, so guidelines document, video and morning questions became our instructional materials. We updated information periodically during ongoing education programs according to the continuous update of infection control guidelines. Nursing department, Medical department and Infection-Control department surveilled every infection control link strictly and at any time to ensure continuous rectification of potential risk factors.

\section{RESULTS}

From January 23 to March 15, 2020, our gastrointestinal surgical ward opened 43 beds, and a total of 192 patients were admitted, 72 patients were operated on, 89 patients received chemotherapy after surgery, and 187 patients were discharged, without any covid-19 case.

\section{DISCUSSION}

Limiting points of entry to control the source of infection, epidemiological investigation are important parts in the infection control. The incubation period for COVID-19 is thought to extend to 14 days, with a median time of 4-5 days from exposure to symptoms onset.11Guan WJ, Ni ZY, Hu Y, et al. Clinical Characteristics of Coronavirus Disease 2019 in China. The New England journal of medicine. 2020.22Li Q, Guan X, Wu P, et al. Early Transmission Dynamics in Wuhan, China, of Novel Coronavirus-Infected Pneumonia. The New England journal of medicine. 2020. 97.5\% of persons with COVID-19 develop symptoms of SARS-CoV-2 infection within 11.5 days.33Lauer SA, Grantz KH, Bi Q, et al. The Incubation Period of Coronavirus Disease 2019 (COVID-19) From Publicly Reported Confirmed Cases: Estimation and Application. Annals of internal medicine. 2020. Therefore, screening everyone entering our ward for COVID-19 symptoms at the admission office and the re-screening at the entrance of our ward, including checking the travel track of all patients and workers, then monitoring daily temperature within 14 days every day44Chinese Society of Cardiology. Chinese Clinical Guidance For COVID-19 Pneumonia Diagnosis and Treatment. China: Chinese Society of Cardiology; 2020 [cited 2020 Apr. 16]. Available from:http://kjfy.meetingchina.org/msite/news/show/cn/3337.html. became the key points of ward management.

The role of the families in improving ill patients' conditions is important. A flexible visiting policy can have a positive effect of the patients condition and eventually their families and help them cope with physiological and emotional disruption.55Khaleghparast S, Joolaee S, Ghanbari B, Maleki M, Peyrovi H, Bahrani N. A Review of Visiting Policies in Intensive Care Units. Glob J Health Sci. 2015;8(6):267-276. In China, if a patient is hospitalized, an escort family will take care of him during his stay and his other family members, relatives, friends and colleagues will continue to visit him, visiting and patients' accompany management become parts of ward management. As a result, visitors would be the potential source of infection, especially those from high-risk areas during the COVID-19 epidemic. Therefore, we re-established and constantly updated policies for visiting and accompany to restrict visitors and control exogenous sources of infection. 
However, the rapidly evolving COVID-19 pandemic, the restriction of visitors and their own diseases lead to the negative psychological state of patients in different degrees.66MA Kai-xuan,ZHANG Yi-de,HOU Tianya,WU Ming-lan,CAI Wen-peng,WEN Tong, Investigation of physical and mental health in isolated people during the outbreak of novel coronavirus pneumonia[J].Chinese Journal of Clinical Medicine,2020,27(1):3640. So, humane care, fixed escort and essential visitation were allowed in our ward. The use of alternative mechanisms for patient and visitor interactions such as video-call applications on cell phones or tablets, call and message have received good results.

Hand is the main transmission route of respiratory infectious diseases, especially the healthcare professionals' hands being the most common transmission route.77Graveto JMGN, Rebola R, Fernandes E, Costa PS. Hand hygiene: nurses' adherence after training. Rev Bras Enferm. 2018;71(3):1189-93. Hand hygiene as a key infection prevention and control intervention is widely accepted as the cornerstone for preventing healthcare-associated infections. The ability of hand hygiene, including hand washing or the use of alcohol-based hand sanitizers to prevent the transmission of pathogens in healthcare settings is related to reductions in the number of viable pathogens that transiently contaminate the hands.88Pidot SJ, Gao W, Buultjens AH, et al. Increasing tolerance of hospital Enterococcus faecium to handwash alcohols. Sci Transl Med. 2018;10(452):eaar6115. Therefore, more attention should be paid to hand hygiene of heathcare personnel in our hospital by offering alcohol-based ABHR with $75 \%$ ethanol which can inactivate viruses that are genetically related to, and with similar physical properties as, the $2019-\mathrm{nCoV}$ at the entrance of inpatient building, ward and rooms.99National Health Committee of the People's Republic of China. Specification of hand hygiene for healthcare workers: WS/T 313-2019 [S/OL]. (2019-11-26) [2020-04-07]. http://www.nhc.gov.cn/wjw/s9496/202002/dbd143c44abd4de8b59a235feef7d75e/files/6a3e2bf3d82b4ee8a718dbfc3cde8338.p

Minimizing chance for exposures to occupational infections is a fundamental method of protecting healthcare personnel, and personal protective equipment(PPE) is an effective control. In times of shortages, specifically facemasks, N95 respirators and gowns at the begining of COVID-19 outbreak, we made strict system and PPE usage standard so as to protect healthcare personnel and avoid the waste of PPE. Appropriate PPE and alternatives were provided depends on their job duties. Nurses and physicians would wear facemasks for part of the day when not engaged in direct patient care activities, only switching to higher level respirator when PPE is required and reusable PPE must be properly cleaned, decontaminated, and maintained after and between uses.

Supervision is the most cost-effective and effective means to ensure that policies and measures are effectively implemented. Timely and periodical record of the implementation of the air and surface disinfection is a convenient way.

As the COVID-19 pandemic progresses, staffing shortages occurred due to the medical staff support for the prevention and control of the COVID-19 epidemic in hubei province. Strategies to mitigate staffing shortages are available, including training and education, material and emotional support to assist HCP with anxiety and stress, flexible shift according to the workload and difficulty of the work.

\section{CONCLUSION}

Epidemiological investigation, personnel management, disinfection isolation, training and education and surveillance form a quality management circle of ward managetment based on closed-loop management.11Robert S. Kaplan, David P. Norton. Closed-loop management: from strategy to operations [J]. Harvard business review, 2008;(2): 46-60. By optimizing the personnel management and supplies management, as well as infection control guidelines, we have controlled the source of infection, cut off the transmission route and ensured the normal operation of the ward without any covid-19 case. Only with positive response, can COVID-19 prevention and medical work be in an orderly manner.

\section{REFERENCE}

1 World Health Organization. WHO Director-General's opening remarks at the media briefing on COVID-19 -11 March 2020. Geneva: World Health Organization; 2020 
2 Wang D, Hu B, Hu C, et al. Clinical Characteristics of 138 Hospitalized Patients With 2019 Novel Coronavirus-Infected Pneumonia in Wuhan, China, JAMA. 2020;e201585. doi:10.1001/jama.2020.1585

3 Standing Committee of the National People's Congress. Law on prevention and control of infectious diseases of the People's Republic of China (2013-6-29, Revised). China, Standing Committee of the National People's Congress, June 29, 2013. http://www.gov.cn/banshi/2005- 08/01/content_19023.htm.

4 National Health Committee of the People's Republic of China. Chinese Clinical guideline for COVID-19 diagnosis and treatment (5th Edition). China, National Health Committee, February 4, 2020.http://www.nhc.gov.cn/yzygj/s76

5 National Health Committee of the People's Republic of China. Guideline of prevention and control of COVID-19 in medical institution (1st Edition). China, National Health Committee, January 22, 2020. http://www.nhc.gov.cn/yzygj/s7659/202001/b91fdab7c304431eb082d67847d27e14.shtml.

6 National Health Committee of People's Republic of China. Regulation for prevention and control of healthcare associated infection of airborne transmission disease in healthcare facilities. December 27, 2016. http://www.nhc.gov.cn/wjw/s9496/201701/7e0e8fc6725843aabba8f841f2f585d2.shtml.

7 Guan WJ, Ni ZY, Hu Y, et al. Clinical Characteristics of Coronavirus Disease 2019 in China [published online ahead of print, 2020 Feb 28]. N Engl J Med. 2020;NEJMoa2002032. doi:10.1056/NEJMoa2002032

8 Li Q, Guan X, Wu P, et al. Early Transmission Dynamics in Wuhan, China, of Novel Coronavirus-Infected Pneumonia. N Engl J Med. 2020;382(13):1199-1207. doi:10.1056/NEJMoa2001316

9 Lauer SA, Grantz KH, Bi Q, et al. The Incubation Period of Coronavirus Disease 2019 (COVID-19) From Publicly Reported Confirmed Cases: Estimation and Application [published online ahead of print, 2020 Mar 10]. Ann Intern Med. 2020;M20-0504. doi:10.7326/M20-0504

10 Chinese Society of Cardiology. Chinese Clinical Guidance For COVID-19 Pneumonia Diagnosis and Treatment. China: Chinese Society of Cardiology; 2020 [cited 2020 Apr. 16]. Available from:http://kjfy.meetingchina.org/msite/ne

11 Khaleghparast S, Joolaee S, Ghanbari B, Maleki M, Peyrovi H, Bahrani N. A Review of Visiting Policies in Intensive Care Units. Glob J Health Sci. 2015;8(6):267-276.

12 Ma KX, Zhang YD, Hou TY, et al. Investigation of physical and mental health in isolated people during the outbreak of novel coronavirus pneumonia.Chin J Clin Med,2020;27(1):36-40.

13 Graveto JMGN, Rebola R, Fernandes E, Costa PS. Hand hygiene: nurses' adherence after training. Rev Bras Enferm. 2018;71(3):1189-93.

14 Pidot SJ, Gao W, Buultjens AH, et al. Increasing tolerance of hospital Enterococcus faecium to handwash alcohols. Sci Transl Med. 2018;10(452):eaar6115.

15 National Health Committee of the People's Republic of China. Specification of hand hygiene for healthcare workers: WS/T 313-2019 [S/OL]. (2019-11-26) [2020-04-07]. http://www.nhc.gov.cn/wjw/s9496/202002/dbd143c44abd4de8b

16 Robert S. Kaplan, David P. Norton. Closed-loop management: from strategy to operations. Harvard business review, 2008;(2): 46-60. 